hep-th/0012067

UFES-DF-OP2000/4

CBPF-NF-076/00

ICEN-PS-00/7

\title{
Finiteness of PST self-dual models
}

\author{
Oswaldo M. Del Cima ${ }^{(\mathrm{a})}$, Olivier Piguet ${ }^{(\mathrm{b}) 1}$ and Marcelo S. Sarandy ${ }^{(\mathrm{c})} \mathrm{H}^{\mathrm{h}}$ \\ (a) Universidade Católica de Petrópolis (UCP), \\ Grupo de Física Teórica, \\ Rua Barão do Amazonas 124 - 25685-070 - Petrópolis - RJ - Brazil. \\ (b) Universidade Federal do Espírito Santo (UFES), \\ Departamento de Física, \\ Campus Universitário de Goiabeiras - 29060-900 - Vitória - ES - Brazil. \\ (c) Centro Brasileiro de Pesquisas Físicas (CBPF), \\ Coordenação de Teoria de Campos e Partículas (CCP), \\ Rua Dr. Xavier Sigaud 150 - 22290-180 - Rio de Janeiro - RJ - Brazil. \\ E-mails:delcima@gft.ucp.br, piguet@cce.ufes.br, sarandy@cbpf.br
}

\begin{abstract}
The Pasti-Sorokin-Tonin model for describing chiral forms is considered at the quantum level. We study the ultraviolet and infrared behaviour of the model in two, four and six dimensions in the framework of algebraic renormalization. The absence of anomalies, as well as the finiteness, up to non-physical renormalizations, are shown in all dimensions analyzed.
\end{abstract}

\section{Introduction}

Antisymmetric tensor fields with self-dual field strengths, also called chiral forms or chiral bosons, appear in a wide context in theoretical physics, mainly related to superstring

\footnotetext{
${ }^{1}$ Supported by the Conselho Nacional de Desenvolvimento Científico e Tecnológico CNPq - Brazil.
} 
theories [1], M-theory five-branes [2], chiral supergravity [3] and also in connection with fractional quantum Hall effect [4] and statistical systems involving the Coulomb Gas [0].

Various formulations, non-manifestly [6]-8] and manifestly Lorentz covariant [9]-14] have been proposed to describe chiral bosons. Among the manifestly Lorentz covariant versions, it can be pointed out the Siegel model [9], which imposes the square of the self-duality condition. It is affected by a gauge anomaly, what is extensively discussed in [15]. In addition, one can still mention the two-dimensional covariant model proposed by McClaim-Wu-Yu [10] and Wotzasek [11], which introduces an infinite tower of auxiliary fields. This formulation has been generalized for higher dimensions in [12].

Recently, a remarkable Lorentz covariant approach to describe chiral bosons, proposed by Pasti, Sorokin and Tonin (PST) [13, 14, has been a subject of intensive research [16, 17, 18]. It introduces, in contrast to [10, 11, 12, only one scalar auxiliary field, but in a non-polynomial way. The analysis is performed in Minkowski space-times with even dimension[2, as usual in self-dual models. However, if the dimension is $D=4 p$, with p integer, it is not possible to define a non-vanishing $2 p$-form self-dual field-strength. So, in this case, a modified version of this approach has been defined. In particular, the PST model in four dimensions has been discussed [13], which describes a manifestly Lorentz and duality symmetric Maxwell electromagnetism. It is based on the duality symmetric - but non-manifestly Lorentz invariant - Schwarz-Sen model [8] and introduces, besides a non-polynomial dependence on a scalar auxiliary field, the presence of an auxiliary gauge potential. Then the self-duality property is replaced by a duality relation between the two field-strengths of the theory.

The PST mechanism has been used, among other things, to construct a covariant effective action for the M-theory five-brane [17], covariant actions for chiral supersymmetric bosons [18] and to rederive the gravitational anomaly for the chiral bosons [16].

Our purpose in this work is to investigate the quantum behaviour of the PST models in the framework of the algebraic renormalization [20], analyzing in detail the issue of the anomalies and the stability of the classical action under radiative corrections. The absence of gauge anomaly has already been pointed out in two dimensions [14, 16]. We give an algebraic proof of this result, including also the cases of four and six dimensions. Moreover we show the finiteness of the PST models, up to non-physical renormalizations, in all dimensions analyzed.

The paper is organized as follows. In section 2 we describe the PST model in $\mathrm{D}=2$ at the classical and quantum levels, discussing its infrared and ultraviolet properties, showing the absence of gauge anomaly as well as the finiteness. In section 3 , we extend these results to the PST formulation in $\mathrm{D}=6$. In Section 4 we discuss the modified version of the PST approach in $\mathrm{D}=4$, for which we show that the same results hold. In section 5

\footnotetext{
${ }^{2}$ See also the discussion about self-dual models defined in odd and even dimensional space-times by the mechanism of dual projection [19].
} 
we summarize our main results, presenting our conclusions.

\section{The PST formulation in $\mathrm{D}=2$}

\subsection{The classical aspects}

The classical PST action in $\mathrm{D}=2$ is defined as 13

$$
\Sigma_{\mathrm{inv}}=\int d^{2} x \frac{1}{2}\left(-F^{\mu} F_{\mu}+\frac{1}{(\partial a)^{2}}\left(\partial^{\mu} a \mathcal{F}_{\mu}\right)^{2}\right),
$$

where $F_{\mu} \equiv \partial_{\mu} \phi$ is the field strength of the scalar field $\phi, \mathcal{F}_{\mu} \equiv\left(\eta_{\mu \nu}-\epsilon_{\mu \nu}\right) \partial^{\nu} \phi=-\epsilon_{\mu \nu} \mathcal{F}^{\nu}$ and $a$ is a scalar field?.

The action (2.1) is invariant under the following transformations :

$$
\begin{aligned}
& \delta_{\alpha} \phi=\alpha(x) B, \quad \delta_{\alpha} a=\alpha(x), \quad \text { with } B \equiv \frac{\mathcal{F}_{\mu} \partial^{\mu} a}{(\partial a)^{2}} ; \\
& \delta_{\epsilon} \phi=f(a), \quad \delta_{\epsilon} a=0, \quad \text { with } f(a) \text { an arbitrary function of } a .
\end{aligned}
$$

Actually the transformation (2.3) corresponds to an infinite set of global symmetries. Using this invariance and the equation of motion for $\phi(x)$ we can get the self-duality condition $\mathcal{F}_{\mu}=0$ [21], which implies that $\phi(x)$ is a chiral scalar. By means of the local symmetry (2.2) we can choose $\partial_{\mu} a=\delta_{\mu}^{0}$, getting the non-manifestly Lorentz covariant Floreanini-Jackiw model [6]. However, as our result will show, the symmetry (2.2), together just with the usual power-counting bounds for the dimension, is sufficient to fix the theory (up to field redefinitions) in the quantum regime as well as in the classical approximation. Hence we shall not need to include the invariance under (2.3) among our basic requirements. The same observation holds for the higher dimensional models studied in the next sections.

In order to quantize the theory we must first determine the vacuum field configuration. The auxiliary field $a$ enters in the action in a non-polynomial way and, to avoid a singularity, the condition $\partial^{\mu} a \partial_{\mu} a \neq 0$ has to be imposed. So $a(x)$ is split as

$$
a(x)=\bar{a}(x)+a^{\prime}(x),
$$

where $\bar{a}(x)$ is the vacuum expectation value of $a(x)$ and $a^{\prime}(x)$ is its quantum fluctuation.

\footnotetext{
${ }^{3}$ We will work in Minkowski space-time with metric $\operatorname{diag}(1,-1, \ldots,-1)$. The Levi-Civita tensor $\epsilon_{\mu_{1} \ldots \mu_{D}}$ is defined by $\epsilon^{01 \ldots D-1}=1=(-1)^{D-1} \epsilon_{01 \ldots D-1}$.
} 
We define the BRST transformations corresponding to the gauge symmetry (2.2) as

$$
\begin{aligned}
& s \phi=c B \\
& s a^{\prime}=c \\
& s c=0 .
\end{aligned}
$$

where $c$ is the Faddeev-Popov ghost.

Moreover we have

$$
s \Sigma_{\mathrm{inv}}=0 \quad, \quad s^{2}=0
$$

In order to implement the gauge-fixing of the local symmetry (2.2) let us introduce a Lagrange multiplier field $\pi$ and an antighost field $\bar{c}$. The gauge-fixing action is given by

$$
\Sigma_{\mathrm{gf}}=s \int d^{2} x \bar{c} a^{\prime}=\int d^{2} x\left(\pi a^{\prime}-\bar{c} c\right)
$$

with

$$
s \bar{c}=\pi \quad, \quad s \pi=0 .
$$

In view of expressing the BRST invariance in a functional way by a Slavnov-Taylor identity we add to the action a term coupling an external field $\phi^{*}$ ("antifield" [22]) to the BRST variation of $\phi$, which is non-linear and hence subjected to possible renormalization:

$$
\Sigma_{\text {ext }}=\int d^{2} x \phi^{*} s \phi=\int d^{2} x \phi^{*} c B
$$

An important point to be noticed concerns the infrared (IR) behaviour of the theory, which can be analyzed from the free propagators, listed below in momentum-spacef (considering $\partial \bar{a}$ as a constant):

$$
\begin{aligned}
& \langle\phi \phi\rangle=\left(-p^{2}+\frac{\partial^{\mu} \bar{a} \partial^{\nu} \bar{a}}{(\partial \bar{a})^{2}}\left(p_{\mu} p_{\nu}-2 \epsilon_{\nu \gamma} p_{\mu} p^{\gamma}+\epsilon_{\mu \lambda} \epsilon_{\nu \gamma} p^{\lambda} p^{\gamma}\right)\right)^{-1}, \\
& \langle\bar{c} c\rangle=1, \\
& \left\langle a^{\prime} \pi\right\rangle=1 .
\end{aligned}
$$

As we can see the propagator $\langle\phi \phi\rangle$ is not integrable at small momenta. This is a typical behaviour of the massless scalar fields in two space-time dimensions. So we must regularize the ill defined propagator at long distances and, in order to do so, we introduce a regulator mass 23] such that

$$
\langle\phi \phi\rangle=\left(-p^{2}+\frac{\partial^{\mu} \bar{a} \partial^{\nu} \bar{a}}{(\partial \bar{a})^{2}}\left(p_{\mu} p_{\nu}-2 \epsilon_{\nu \gamma} p_{\mu} p^{\gamma}+\epsilon_{\mu \lambda} \epsilon_{\nu \gamma} p^{\lambda} p^{\gamma}\right)+m_{\phi}^{2}\right)^{-1}
$$

\footnotetext{
${ }^{4}$ Using the abbreviation notation $\langle\ldots\rangle \equiv\langle 0|T \ldots| 0\rangle_{\text {free, }}$ where $T$ is the time ordering operator.
} 
where $m_{\phi}^{2}$ is a regulator mass, giving a well defined behaviour for the propagator in the IR limit.

Such a mass may be introduced, formally keeping BRST invariance, through the mass action

$$
\Sigma_{\mathrm{m}}=s \int d^{2} x \frac{1}{2} \tau_{2} \phi^{2}=\int d^{2} x\left(\frac{1}{2}\left(\tau_{1}+m_{\phi}^{2}\right) \phi^{2}-\tau_{2} \phi c B\right),
$$

where $\tau_{1}$ and $\tau_{2}$ are new external fields with the following BRST transformations :

$$
s \tau_{2}=\tau_{1}+m_{\phi}^{2} \quad, \quad s \tau_{1}=0 .
$$

So, the total classical action for the PST model in $\mathrm{D}=2$,

$$
\Sigma=\Sigma_{\text {inv }}+\Sigma_{\text {gf }}+\Sigma_{\text {ext }}+\Sigma_{\mathrm{m}}
$$

is invariant under BRST transformations, what is expressed in a functional way by the Slavnov-Taylor identity

$$
\mathcal{S}(\Sigma)=\int d^{2} x\left(\frac{\delta \Sigma}{\delta \phi^{*}} \frac{\delta \Sigma}{\delta \phi}+c \frac{\delta \Sigma}{\delta a^{\prime}}+\pi \frac{\delta \Sigma}{\delta \bar{c}}+\left(\tau_{1}+m_{\phi}^{2}\right) \frac{\delta \Sigma}{\delta \tau_{2}}\right)=0 .
$$

From (2.17) we define the linearized Slavnov-Taylor operator as below

$$
\mathcal{S}_{\Sigma}=\int d^{2} x\left(\frac{\delta \Sigma}{\delta \phi^{*}} \frac{\delta}{\delta \phi}+\frac{\delta \Sigma}{\delta \phi} \frac{\delta}{\delta \phi^{*}}+c \frac{\delta}{\delta a^{\prime}}+\pi \frac{\delta}{\delta \bar{c}}+\left(\tau_{1}+m_{\phi}^{2}\right) \frac{\delta}{\delta \tau_{2}}\right)
$$

For any functional $\gamma$ it can be shown to hold $\mathcal{S}_{\gamma} \mathcal{S}(\gamma)=0$. Moreover taking into account the Slavnov-Taylor identity (2.17), the linearized Slavnov-Taylor operator turns out to be nilpotent :

$$
\mathcal{S}_{\Sigma} \mathcal{S}_{\Sigma}=0
$$

The ultraviolet (UV) and infrared (IR) dimensions, as well as the Faddeev-Popov charge of all fields are displayed in Table 1.

\begin{tabular}{|c||c|c|c|c|c|c|c|c|c|}
\hline & $\phi$ & $a^{\prime}$ & $c$ & $\pi$ & $\bar{c}$ & $\phi^{*}$ & $\tau_{1}$ & $\tau_{2}$ & $s$ \\
\hline \hline$d$ & 0 & -1 & -1 & 3 & 3 & 2 & 2 & 2 & 0 \\
\hline$r$ & 1 & -1 & -1 & 3 & 3 & 2 & 2 & 2 & 0 \\
\hline$\Phi \Pi$ & 0 & 0 & 1 & 0 & -1 & -1 & 0 & -1 & 1 \\
\hline
\end{tabular}

Table 1: UV and IR dimensions $d$ and $r$, ghost number $\Phi \Pi$.

Before discussing the quantum behaviour of the model let us note important constraints, besides the Slavnov-Taylor identity, obeyed by the total classical action (2.16). The gauge condition and the ghost equation read

$$
\frac{\delta \Sigma}{\delta \pi}=a^{\prime} \quad, \quad \frac{\delta \Sigma}{\delta \bar{c}}=-c
$$


The dependence of the total classical action (2.16) on $a^{\prime}$ and $\bar{a}$ is essentially through the combination $a^{\prime}+\bar{a}$, what is expressed by the following constraint :

$$
\left(\frac{\delta}{\delta a^{\prime}}-\frac{\delta}{\delta \bar{a}}\right) \Sigma=\pi .
$$

where the r.h.s. is a classical insertion, i.e., a linear term in the quantum fields which thus will not get renormalized.

Moreover, important constraints are given by the integrated equations of motion of $a^{\prime}$ and $\phi$, which imply that the total classical action depends essentially on the derivatives of these fields. These equations are given by

$$
\begin{aligned}
& \int d^{2} x \frac{\delta \Sigma}{\delta a^{\prime}}=\int d^{2} x \pi \\
& \int d^{2} x\left(\frac{\delta}{\delta \phi}+\tau_{2} \frac{\delta}{\delta \phi^{*}}\right) \Sigma=\int d^{2} x\left(\tau_{1}+m_{\phi}^{2}\right) \phi .
\end{aligned}
$$

where the r.h.s. of these equations are also classical insertions.

\subsection{The quantum aspects}

This subsection is devoted to study the possibility of implementing the Slavnov-Taylor identity at the quantum level and the stability of the classical action under quantum corrections. This amounts to study all possible anomalies and invariant counterterms in the total action.

First, it is important to mention that there is no problem in the quantum extension of constraints of the type $(2.20-2.23$ [20].

In order to show that the Slavnov-Taylor identity can be implemented at the quantum level we will start by applying the quantum action principle (QAP) [20, 24], which assures that any possible breaking $\Delta^{(1)}$ of the Slavnov-Taylor identity takes the following form :

$$
\mathcal{S}(\Gamma)=\Delta^{(1)} \cdot \Gamma=\Delta^{(1)}+\mathcal{O}\left(\hbar \Delta^{(1)}\right)
$$

where $\Gamma$ is the vertex functional and $\Delta^{(1)}$ is a local, integrated, Lorentz invariant polynomial of UV dimension $\leq 2$, IR dimension $\geq 2$ and ghost number 1 .

The identity $\mathcal{S}_{\Gamma} \mathcal{S}(\Gamma)=0$ and the fact that $\mathcal{S}_{\Gamma}=\mathcal{S}_{\Sigma}+\mathcal{O}(\hbar)$, lead to the Wess-Zumino consistency condition for the breaking $\Delta^{(1)}$ :

$$
\mathcal{S}_{\Sigma} \Delta^{(1)}=0
$$

which constitutes a cohomology problem. The proof of the absence of anomaly consists in showing that $\Delta^{(1)}$ is in the trivial sector of the cohomology, i.e., $\Delta^{(1)}=\mathcal{S}_{\Sigma} \hat{\Delta}^{(0)}$, where $\hat{\Delta}^{(0)}$ is a local polynomial in the fields, with ghost number 0 , called noninvariant counterterm. 
In order to characterize all possible invariant counterterms $\Delta^{(0)}$, considered as perturbations of the total classical action, we are led to solve again a cohomology equation :

$$
\mathcal{S}_{\Sigma} \Delta^{(0)}=0
$$

where $\Delta^{(0)}$ is a local, integrated, Lorentz invariant polynomial of $\mathrm{UV}$ dimension $\leq 2$, IR dimension $\geq 2$ and ghost number 0 .

Anomalies and counterterms $\Delta^{(G)}$ (where $G=0,1$ denotes the ghost number) have also to obey the conditions :

$$
\begin{aligned}
& \frac{\delta \Delta^{(G)}}{\delta \pi}=0 \quad, \quad \frac{\delta \Delta^{(G)}}{\delta \bar{c}}=0, \\
& \left(\frac{\delta}{\delta a^{\prime}}-\frac{\delta}{\delta \bar{a}}\right) \Delta^{(G)}=0, \\
& \int d^{2} x \frac{\delta \Delta^{(G)}}{\delta a^{\prime}}=0, \\
& \int d^{2} x\left(\frac{\delta}{\delta \phi}+\tau_{2} \frac{\delta}{\delta \phi^{*}}\right) \Delta^{(G)}=0 .
\end{aligned}
$$

which follow from requiring the fulfillment of the constraints $2.20-2.23)$ to all orders.

In order to determine the cohomology of $\mathcal{S}_{\Sigma}$ one introduces the counting operator

$$
\mathcal{N}=\int d^{2} x \sum_{i} \Phi_{i} \frac{\delta}{\delta \Phi_{i}},
$$

with $\Phi_{i}$ running over all fields. So one can decompose $\mathcal{S}_{\Sigma}$ as

$$
\mathcal{S}_{\Sigma}=\mathcal{S}_{\Sigma}^{0}+\mathcal{S}_{\Sigma}^{1}+\ldots
$$

where $\mathcal{S}_{\Sigma}^{n}$ is the contribution of power $n$ in the fields for $\mathcal{S}_{\Sigma}$, defined by means of $\left[\mathcal{N}, \mathcal{S}_{\Sigma}^{n}\right]=$ $n \mathcal{S}_{\Sigma}^{n}$. The $\mathcal{S}_{\Sigma}^{0}$-transformations read

$$
\begin{array}{ll}
\mathcal{S}_{\Sigma}^{0} a^{\prime}=c, & \mathcal{S}_{\Sigma}^{0} c=0, \\
\mathcal{S}_{\Sigma}^{0} \phi=0, & \mathcal{S}_{\Sigma}^{0} \phi^{*}=\left.\frac{\delta \Sigma}{\delta \phi}\right|_{\text {linear approx. }} \\
\mathcal{S}_{\Sigma}^{0} \bar{c}=\pi, & \mathcal{S}_{\Sigma}^{0} \pi=0, \\
\mathcal{S}_{\Sigma}^{0} \tau_{2}=\tau_{1}+m_{\phi}^{2}, & \mathcal{S}_{\Sigma}^{0} \tau_{1}=0 .
\end{array}
$$

According to a general theorem [20, 25, 26], the cohomology of the complete operator $\mathcal{S}_{\Sigma}$ is isomorphic to a subspace of the cohomology of the operator $\mathcal{S}_{\Sigma}^{0}$. Thus we will first focus on the operator $\mathcal{S}_{\Sigma}^{0}$. Studying the most general dependence of the cohomology on the antifield $\phi^{*}$ we write down

$$
\Delta^{(G)}=\int d^{2} x \phi^{*} Z^{(G+1)}+\text { terms independent of the antifield . }
$$


where $Z^{(G+1)}$ is an arbitrary local, non-integrated, Lorentz invariant polynomial of UV dimension $\leq 0$, IR dimension $\geq 0$ and ghost number $G+1$ depending on all fields except $\phi^{*}$. Hence from (2.25) and (2.26), at lowest order in $n$, we have:

$$
\begin{aligned}
\mathcal{S}_{\Sigma}^{0} \Delta^{(G)} & =0 \\
& =-\int d^{2} x \phi^{*} \mathcal{S}_{\Sigma}^{0} Z^{(G+1)}+\text { terms independent of the antifield }
\end{aligned}
$$

From (2.32) we get a new (local) cohomology problem:

$$
\mathcal{S}_{\Sigma}^{0} Z^{(G+1)}(x)=0
$$

We observe in (2.30) that various fields appear as $\operatorname{BRST}$ doublets $\left(\varphi_{1}, \varphi_{2}=\mathcal{S}_{\Sigma}^{0} \varphi_{1}\right)$, namely $(\bar{c}, \pi),\left(\tau_{2}, \tau_{1}+m_{\phi}^{2}\right),\left(a^{\prime}, c\right)$, as well all their derivatives. As it is well known [20, 25, such doublets belong to the trivial sector of the cohomology.

Since $c$ and its derivatives in particular are in doublets, there are no fields with positive ghost number in the non-trivial sector of the cohomology, which thus is empty for $G=0,1$.

Let us now analyze the terms independent of $\phi^{*}$. This means to study

$$
\mathcal{S}_{\Sigma}^{0} \int d^{2} x Q^{(G)}=0
$$

where $Q^{(G)}$ has UV dimension $\leq 2$, IR dimension $\geq 2$ and ghost number $G=1$ for anomalies and $G=0$ for the invariant counterterms.

From (2.34) and the algebraic Poincaré lemma [27] one deduces the set of descent equations

$$
\begin{aligned}
& \mathcal{S}_{\Sigma}^{0} Q^{(G)}=\partial^{\mu} Q_{\mu}^{(G+1)}, \\
& \mathcal{S}_{\Sigma}^{0} Q_{\mu}^{(G+1)}=\partial^{\nu} Q_{[\mu \nu]}^{(G+2)}, \\
& \mathcal{S}_{\Sigma}^{0} Q_{[\mu \nu]}^{(G+2)}=0
\end{aligned}
$$

There is no non-trivial solution with ghost number $G=1$, what means that the cohomology of $\mathcal{S}_{\Sigma}$ with $G=1$ is empty and the model is anomaly-free.

Concerning the possible invariant counterterms $(G=0)$ we get that the most general non-trivial solution for the top level of the descent equations is given by

$$
Q^{(0)}=\Omega(\phi, \bar{a})
$$

where $\Omega(\phi, \bar{a})$ is an arbitrary polynomial of $\phi$ - with coefficients as arbitrary functions of $\bar{a}-$ with UV dimension $\leq 2$, IR dimension $\geq 2$ and ghost number 0 .

In order to determine the cohomology of the full operator $\mathcal{S}_{\Sigma}$, we must first complete the solution (2.36) to an invariant of $\mathcal{S}_{\Sigma}$, imposing the constraints (2.27). So we get that the most general non-trivial counterterm can be at most the invariant action (2.1) :

$$
\int d^{2} x Q^{(0)}=\Sigma_{\text {inv }}
$$


However $\Sigma_{\text {inv }}$ is trivial :

$$
\Sigma_{\mathrm{inv}}=\frac{1}{2} \mathcal{S}_{\Sigma} \int d^{2} x \phi \phi^{*}-\Sigma_{\mathrm{m}}
$$

since the mass term $\Sigma_{\mathrm{m}}$ is trivial, according to (2.14). Therefore, the cohomology of $\mathcal{S}_{\Sigma}$ in the sector $G=0$ is empty and the PST model in $D=2$ is finite, up to possible nonphysical renormalizations of the field amplitudes which correspond to the trivial solution of the cohomology equation (2.26).

It is important to mention that it remains the question of the massless limit $m_{\phi} \rightarrow 0$, since $m_{\phi}$ is not a physical mass and has been introduced just as an infrared cut-off. This kind of discussion can be found in [26] in the context of the two dimensional non-linear sigma model and in 23] in connection with the BF model.

\section{Finiteness of the $\mathrm{D}=6$ model}

\subsection{The classical model}

The extension of the PST action to dimension $\mathrm{D}=6$ is given by [13, 14]

$$
\Sigma_{\mathrm{inv}}=\int d^{6} x\left(-\frac{1}{6} F^{\mu \nu \rho} F_{\mu \nu \rho}+\frac{1}{2(\partial a)^{2}} \partial^{\mu} a \mathcal{F}_{\mu \nu \rho} \mathcal{F}^{\nu \rho \lambda} \partial_{\lambda} a\right),
$$

where $F_{\mu \nu \rho} \equiv \partial_{\mu} A_{\nu \rho}+\partial_{\rho} A_{\mu \nu}+\partial_{\nu} A_{\rho \mu}$ is the field strength of the tensorial field $A_{\mu \nu}$,

$$
\mathcal{F}_{\mu \nu \rho} \equiv F_{\mu \nu \rho}-\frac{1}{3 !} \epsilon_{\mu \nu \rho \sigma \epsilon \delta} F^{\sigma \epsilon \delta}=-\frac{1}{3 !} \epsilon_{\mu \nu \rho \sigma \epsilon \delta} \mathcal{F}^{\sigma \epsilon \delta}
$$

and $a$ is a scalar auxiliary field.

The action (3.1) is invariant under the following set of four transformations:

$$
\begin{array}{lll}
\delta_{I} A_{\mu \nu}=2 \partial_{[\mu} \alpha_{\nu]}=\partial_{\mu} \alpha_{\nu}-\partial_{\nu} \alpha_{\mu}, & \delta_{I} a=0, & \\
\delta_{I I} A_{\mu \nu}=2 \phi_{[\mu} \partial_{\nu]} a=\phi_{\mu} \partial_{\nu} a-\phi_{\nu} \partial_{\mu} a, & \delta_{I I} a=0, & \\
\delta_{I I I} A_{\mu \nu}=\beta B_{\mu \nu}, & \delta_{I I I} a=\beta, & \text { with } B_{\mu \nu} \equiv \frac{\mathcal{F}_{\mu \nu \rho} \partial^{\rho} a}{(\partial a)^{2}}, \\
\delta_{I V} A_{\mu \nu}=f_{\mu \nu}(a), & \delta_{I V} a=0, & \text { with } f_{\mu \nu}(a) \text { an arbitrary } \\
& & \text { function of } a,
\end{array}
$$

where $\alpha_{\mu}, \phi_{\mu}$ and $\beta$ are the infinitesimal parameters of the transformations.

As in $\mathrm{D}=2$ we must require $\partial^{\mu} a \partial_{\mu} a \neq 0$ and so split $a(x)$ in $\bar{a}(x)$ and $a^{\prime}(x)$ according to $(2.4)$. 
The BRST transformations, arising from the local symmetries of the set (3.3) are given by

$$
\begin{aligned}
& s A_{\mu \nu}=2 \partial_{[\mu} \alpha_{\nu]}+2 \phi_{[\mu} \partial_{\nu]} a+\beta B_{\mu \nu}, \\
& s a^{\prime}=\beta, \\
& s \beta=0, \\
& s \alpha_{\mu}=\partial_{\mu} \alpha+\partial_{\mu} a \omega-\phi_{\mu} \beta, \\
& s \phi_{\mu}=-\partial_{\mu} \omega-\partial_{\mu} a \phi+2 \partial_{[\mu} \phi_{\nu]} \frac{\partial^{\nu} a \beta}{(\partial a)^{2}}-B_{\mu \nu} \frac{\beta \partial^{\nu} \beta}{(\partial a)^{2}}, \\
& s \alpha=-\omega \beta, \\
& s \omega=-\phi \beta, \\
& s \phi=-B_{\mu \nu} \frac{\beta \partial^{\mu} \beta \partial^{\nu} \beta}{(\partial a)^{4}}+2 \frac{\partial^{\mu} a}{(\partial a)^{4}} \partial_{[\mu} \phi_{\nu]} \beta \partial^{\nu} \beta+\frac{\partial^{\mu} a}{(\partial a)^{2}} \partial_{\mu} \phi \beta .
\end{aligned}
$$

where $\alpha_{\mu}, \phi_{\mu}$ and $\beta$ are now anticommuting ghosts with ghost number one. The fields $\alpha, \omega$ and $\phi$ are commuting ghosts with ghost number two, which were introduced to fix the residual degrees of freedom coming from the reducible symmetries (the first two symmetries of the set (3.3)). The latter have indeed three zero modes: $\alpha_{\mu}=\partial_{\mu} X$; $\phi_{\mu}=\partial_{\mu} a Y ; \alpha_{\mu}=\partial_{\mu} a Z, \phi_{\mu}=-\partial_{\mu} Z$.

In addition we have

$$
s \Sigma_{\mathrm{inv}}=0, s^{2}=0 .
$$

Following the Batalin-Vilkovisky prescription [22] for theories with reducible symmetries we implement the gauge-fixing action

$$
\begin{gathered}
\Sigma_{\mathrm{gf}}=s \int d^{6} x\left(\bar{\alpha}^{\mu} \partial^{\nu} A_{\mu \nu}+\bar{\phi}^{\mu} A_{\mu \nu} \partial^{\nu} a+\bar{\phi}^{\prime} \partial^{\mu} a \phi_{\mu}+\bar{\alpha} \partial^{\mu} \alpha_{\mu}+\right. \\
\left.+\bar{\phi} \partial^{\mu} \phi_{\mu}+\bar{\beta} a^{\prime}+\bar{\alpha}^{\mu} \partial_{\mu} \bar{c}+\bar{\phi}^{\mu} \partial_{\mu} a \bar{c}^{\prime}\right)
\end{gathered}
$$

where we have introduced the antighosts $\bar{\alpha}^{\mu}, \bar{\phi}^{\mu}, \bar{\alpha}, \bar{\phi}^{\prime}, \bar{\phi}$ and $\bar{\beta}$, the Lagrange multipliers $\pi^{\mu}, \rho^{\mu}, \pi, \rho^{\prime}, \rho$ and $b$ and the extra pairs of fields $(\bar{c}, \lambda)$ and $\left(\bar{c}^{\prime}, \lambda^{\prime}\right)$ transforming as

$$
s \bar{C}=\Pi, \quad s \Pi=0,
$$

with $\bar{C}=\left(\bar{\alpha}^{\mu}, \bar{\phi}^{\mu}, \bar{\alpha}, \bar{\phi}^{\prime}, \bar{\phi}, \bar{\beta}, \bar{c}, \bar{c}^{\prime}\right)$ and $\Pi=\left(\pi^{\mu}, \rho^{\mu}, \pi, \rho^{\prime}, \rho, b, \lambda, \lambda^{\prime}\right)$.

Differently from the case $\mathrm{D}=2$ there is no problem in the infrared limit because all the propagators turn out to be integrable at small momenta in $\mathrm{D}=6$. The UV dimensions and ghost numbers of all fields introduced so far are shown in Table 2 and Table 3.

In order to get the Slavnov-Taylor identity we introduce the external field action

$$
\Sigma_{\mathrm{ext}}=\int d^{6} x\left(\frac{1}{2} A_{\mu \nu}^{*} s A^{\mu \nu}+\alpha_{\mu}^{*} s \alpha^{\mu}+\phi_{\mu}^{*} s \phi^{\mu}+\alpha^{*} s \alpha+\omega^{*} s \omega+\phi^{*} s \phi\right),
$$




\begin{tabular}{|c||c|c|c|c|c|c|c|c|c|}
\hline & $A_{\mu \nu}$ & $a^{\prime}$ & $\alpha_{\mu}$ & $\phi_{\mu}$ & $\beta$ & $\alpha$ & $\omega$ & $\phi$ & $s$ \\
\hline \hline$d$ & 2 & -1 & 1 & 2 & -1 & 0 & 1 & 2 & 0 \\
\hline$\Phi \Pi$ & 0 & 0 & 1 & 1 & 1 & 2 & 2 & 2 & 1 \\
\hline
\end{tabular}

Table 2: dimension $d$ and ghost number $\Phi \Pi$.

\begin{tabular}{|c||c|c|c|c|c|c|c|c|c|c|c|c|c|c|c|c|}
\hline & $\bar{\alpha}^{\mu}$ & $\pi^{\mu}$ & $\bar{\phi}^{\mu}$ & $\rho^{\mu}$ & $\bar{\alpha}$ & $\pi$ & $\bar{\phi}^{\prime}$ & $\rho^{\prime}$ & $\bar{\phi}$ & $\rho$ & $\bar{\beta}$ & $b$ & $\bar{c}$ & $\lambda$ & $\bar{c}^{\prime}$ & $\lambda^{\prime}$ \\
\hline \hline$d$ & 3 & 3 & 4 & 4 & 4 & 4 & 4 & 4 & 3 & 3 & 7 & 7 & 2 & 2 & 2 & 2 \\
\hline$\Phi \Pi$ & -1 & 0 & -1 & 0 & -2 & -1 & -3 & -2 & -2 & -1 & -1 & 0 & 0 & 1 & 0 & 1 \\
\hline
\end{tabular}

Table 3: dimension $d$ and ghost number $\Phi \Pi$.

where $A_{\mu \nu}^{*}, \alpha_{\mu}^{*}, \phi_{\mu}^{*}, \alpha^{*}, \omega^{*}$ and $\phi^{*}$ are external sources, with UV dimensions and ghost numbers given in Table 4 .

So, the total action is

$$
\Sigma=\Sigma_{\mathrm{inv}}+\Sigma_{\mathrm{gf}}+\Sigma_{\mathrm{ext}}
$$

and the Slavnov-Taylor identity is written down as

$$
\begin{aligned}
\mathcal{S}(\Sigma)= & \int d^{6} x\left(\frac{1}{2} \frac{\delta \Sigma}{\delta A_{\mu \nu}^{*}} \frac{\delta \Sigma}{\delta A^{\mu \nu}}+\frac{\delta \Sigma}{\delta \alpha_{\mu}^{*}} \frac{\delta \Sigma}{\delta \alpha^{\mu}}+\frac{\delta \Sigma}{\delta \phi_{\mu}^{*}} \frac{\delta \Sigma}{\delta \phi^{\mu}}+\frac{\delta \Sigma}{\delta \alpha^{*}} \frac{\delta \Sigma}{\delta \alpha}+\frac{\delta \Sigma}{\delta \omega^{*}} \frac{\delta \Sigma}{\delta \omega}+\frac{\delta \Sigma}{\delta \phi^{*}} \frac{\delta \Sigma}{\delta \phi}+\right. \\
& \left.+\beta \frac{\delta \Sigma}{\delta a^{\prime}}+\pi^{\mu} \frac{\delta \Sigma}{\delta \bar{\alpha}^{\mu}}+\rho^{\mu} \frac{\delta \Sigma}{\delta \bar{\phi}^{\mu}}+\pi \frac{\delta \Sigma}{\delta \bar{\alpha}}+\rho^{\prime} \frac{\delta \Sigma}{\delta \bar{\phi}^{\prime}}+\rho \frac{\delta \Sigma}{\delta \bar{\phi}}+b \frac{\delta \Sigma}{\delta \bar{\beta}}+\lambda \frac{\delta \Sigma}{\delta \bar{c}}+\lambda^{\prime} \frac{\delta \Sigma}{\delta \bar{c}^{\prime}}\right)
\end{aligned}
$$

From the Slavnov-Taylor identity we get the nilpotent linearized Slavnov-Taylor operator

$$
\begin{aligned}
\mathcal{S}_{\Sigma}= & \int d^{6} x\left(\frac{1}{2} \frac{\delta \Sigma}{\delta A_{\mu \nu}^{*}} \frac{\delta}{\delta A^{\mu \nu}}+\frac{1}{2} \frac{\delta \Sigma}{\delta A^{\mu \nu}} \frac{\delta}{\delta A_{\mu \nu}^{*}}+\frac{\delta \Sigma}{\delta \alpha_{\mu}^{*}} \frac{\delta}{\delta \alpha^{\mu}}+\frac{\delta \Sigma}{\delta \alpha^{\mu}} \frac{\delta}{\delta \alpha_{\mu}^{*}}+\frac{\delta \Sigma}{\delta \phi_{\mu}^{*}} \frac{\delta}{\delta \phi^{\mu}}+\right. \\
& +\frac{\delta \Sigma}{\delta \phi^{\mu}} \frac{\delta}{\delta \phi_{\mu}^{*}}+\frac{\delta \Sigma}{\delta \alpha^{*}} \frac{\delta}{\delta \alpha}+\frac{\delta \Sigma}{\delta \alpha} \frac{\delta}{\delta \alpha^{*}}+\frac{\delta \Sigma}{\delta \omega^{*}} \frac{\delta}{\delta \omega}+\frac{\delta \Sigma}{\delta \omega} \frac{\delta}{\delta \omega^{*}}+\frac{\delta \Sigma}{\delta \phi^{*}} \frac{\delta}{\delta \phi}+\frac{\delta \Sigma}{\delta \phi} \frac{\delta}{\delta \phi^{*}}+ \\
& \left.+\beta \frac{\delta}{\delta a^{\prime}}+\pi^{\mu} \frac{\delta}{\delta \bar{\alpha}^{\mu}}+\rho^{\mu} \frac{\delta}{\delta \bar{\phi}^{\mu}}+\pi \frac{\delta}{\delta \bar{\alpha}}+\rho^{\prime} \frac{\delta}{\delta \bar{\phi}^{\prime}}+\rho \frac{\delta}{\delta \bar{\phi}}+b \frac{\delta}{\delta \bar{\beta}}+\lambda \frac{\delta}{\delta \bar{c}}+\lambda^{\prime} \frac{\delta}{\delta \bar{c}^{\prime}}\right)
\end{aligned}
$$

The total classical action (3.9) obeys various constraints which can be extended to the quantum level. Let us establish some of them, which will play a special role. First, the

\begin{tabular}{|c||c|c|c|c|c|c|}
\hline & $A_{\mu \nu}^{*}$ & $\alpha_{\mu}^{*}$ & $\phi_{\mu}^{*}$ & $\alpha^{*}$ & $\omega^{*}$ & $\phi^{*}$ \\
\hline \hline$d$ & 4 & 5 & 4 & 6 & 5 & 4 \\
\hline$\Phi \Pi$ & -1 & -2 & -2 & -3 & -3 & -3 \\
\hline
\end{tabular}

Table 4: dimension $d$ and ghost number $\Phi \Pi$. 
integrated antighost equation for $\alpha^{\mu}$ and the non-integrated antighost equation for $\alpha$ are

$$
\int d^{6} x \frac{\delta \Sigma}{\delta \alpha^{\mu}}=0, \quad \frac{\delta \Sigma}{\delta \alpha}=\partial^{\mu} \partial_{\mu} \bar{\alpha}-\partial^{\mu} \alpha_{\mu}^{*}
$$

The integrated equation of motion for $a^{\prime}$, which expresses, up to a classical breaking, that the action depends on $a^{\prime}$ only through its derivatives, reads

$$
\int d^{6} x \frac{\delta \Sigma}{\delta a^{\prime}}=\int d^{6} x b
$$

Finally, there is a constraint between $a$ and $a^{\prime}$, which is also classically broken and expresses that the action depends essentially on the combination $\bar{a}+a^{\prime}$. It is given by

$$
\left(\frac{\delta}{\delta a^{\prime}}-\frac{\delta}{\delta \bar{a}}\right) \Sigma=b .
$$

\subsection{Absence of anomalies and finiteness}

As it was recalled in Section 2, the possible anomalies and non-trivial invariant counterterms are characterized by the following cohomology problem

$$
\mathcal{S}_{\Sigma} \Delta^{(G)}=0
$$

where $\Delta^{(G)}$ is an integrated, Lorentz invariant, local polynomial of UV dimension 6 and ghost number $G$, with the candidates for anomalies in the sector $G=1$ and the possible invariant counterterms in the sector $G=0$.

According to [20] the constraints (3.12 - 3.14) can be implemented at the quantum level. It follows that $\Delta^{(G)}$ obeys the same constraints, but with their right-hand sides set to zero. Hence, in particular, it will depend on $\alpha_{\mu}$ through its derivatives only, and it will not depend on $\alpha$.

In order to solve (3.15), we expand $\mathcal{S}_{\Sigma}$ according to a counting operator, like (2.28), acting on all fields. So, we can write down the following expansion for $\mathcal{S}_{\Sigma}$ :

$$
\mathcal{S}_{\Sigma}=\overline{\mathcal{S}}_{\Sigma}^{0}+\overline{\mathcal{S}}_{\Sigma}^{1}+\ldots
$$

However, instead of simply studying the cohomology of $\overline{\mathcal{S}}_{\Sigma}^{0}$ as we did in the case $\mathrm{D}=2$, we will introduce two new filtrations, one of them corresponding to an expansion in powers of $\phi$ and the other in terms of $\left(\phi_{\mu}, \omega, \alpha\right)$. Denoting the corresponding $n$-th order parameters as $\tilde{\mathcal{S}_{\Sigma}^{n}}$ and $\mathcal{S}_{\Sigma}^{n}$, respectively, we get

$$
\begin{aligned}
& \overline{\mathcal{S}}_{\Sigma}^{0}=\tilde{\mathcal{S}}_{\Sigma}^{0}+\tilde{\mathcal{S}}_{\Sigma}^{1}, \\
& \tilde{\mathcal{S}}_{\Sigma}^{0}=\mathcal{S}_{\Sigma}^{0}+\mathcal{S}_{\Sigma}^{1},
\end{aligned}
$$


with the expansions stopping in order 1 since $\overline{\mathcal{S}}_{\Sigma}^{0}$ and $\tilde{\mathcal{S}}_{\Sigma}^{0}$ are at most linear in $\phi$ and $\left(\phi_{\mu}\right.$, $\omega, \alpha)$, respectively.

These new filtrations are very suitable, as it will be seen below, since they produce more doublet pairs, all of them being left in the trivial sector of the cohomology.

Our aim now is to show that the cohomology of $\mathcal{S}_{\Sigma}^{0}$ is trivial, which implies that the cohomology of $\mathcal{S}_{\Sigma}$ is empty. The $\mathcal{S}_{\Sigma}^{0}$-transformations are

$$
\begin{array}{ll}
\mathcal{S}_{\Sigma}^{0} A_{\mu \nu}=2 \partial_{[\mu} \alpha_{\nu]}, & \mathcal{S}_{\Sigma}^{0} \alpha_{\mu}=0 \\
\mathcal{S}_{\Sigma}^{0} a^{\prime}=\beta, & \mathcal{S}_{\Sigma}^{0} \beta=0 \\
\mathcal{S}_{\Sigma}^{0} \phi=0, & \mathcal{S}_{\Sigma}^{0} \alpha=0 \\
\mathcal{S}_{\Sigma}^{0} \phi_{\mu}=-\partial_{\mu} \omega, & \mathcal{S}_{\Sigma}^{0} \omega=0 \\
\mathcal{S}_{\Sigma}^{0} \Phi^{*}=\left.\frac{\delta \Sigma}{\delta \Phi}\right|_{\text {order } 0} &
\end{array}
$$

with $\Phi^{*}=\left(A_{\mu \nu}^{*}, \phi_{\mu}^{*}, \alpha_{\mu}^{*}, \alpha^{*}, \omega^{*}, \phi^{*}\right)$ and $\Phi=\left(A_{\mu \nu}, \phi_{\mu}, \alpha_{\mu}, \alpha, \omega, \phi\right)$.

The transformations of the antighosts, Lagrange multipliers and pairs of extra fields $(\bar{c}, \lambda)$ and $\left(\bar{c}^{\prime}, \lambda^{\prime}\right)$ remain identical to their BRST transformations as listed in (3.7).

Besides the doublets involving the antighosts, the Lagrange multipliers and the extra fields, there is a further doublet in (3.18), namely $\left(a^{\prime}, \beta\right)$, which eliminates the possibility of having fields with negative dimension in the cohomology of $\mathcal{S}_{\Sigma}^{0}$. Moreover, in the space of non-integrated local polynomials, $\phi_{\mu}$ and its symmetric derivatives compose doublets with derivatives of $\omega$.

Beginning with the cocycles involving the external fields, we can write down the most general dependence on the latters as

$$
\begin{aligned}
\Delta_{\mathrm{ext}}^{(G)}=\int d^{6} x & \left(\frac{1}{2} A_{\mu \nu}^{*} X^{(G+1) \mu \nu}+\alpha_{\mu}^{*} X^{(G+2) \mu}+\phi_{\mu}^{*} Y^{(G+2) \mu}+\alpha^{*} X^{(G+3)}+\omega^{*} Y^{(G+3)}+\right. \\
& \left.+\phi^{*} Z^{(G+3)}\right)+ \text { terms independent of the antifields. }
\end{aligned}
$$

where $X^{(G+1) \mu \nu}, X^{(G+2) \mu}, Y^{(G+2) \mu}, X^{(G+3)}, Y^{(G+3)}$ and $Z^{(G+3)}$ are arbitrary polynomials independent of the external fields.

Solving the condition of $\mathcal{S}_{\Sigma}^{0}$-invariance of (3.19), we can show, in a similar way as in $D=2$, that the dependence on the external fields is $\mathcal{S}_{\Sigma}^{0}$-trivial.

Once the antifields have been eliminated, the cohomology may depend on the remaining fields which do not belong to doublets. In particular, at the non-integrated level, the cohomology will depend only on $\partial_{[\rho} A_{\mu \nu]}, \partial_{[\mu} \phi_{\nu]}, \phi, \partial_{(\mu} \alpha_{\nu)}$ 甲, their derivatives, and $\omega$.

\footnotetext{
${ }^{5} \partial_{(\mu} \alpha_{\nu)}$ denotes symmetric derivative in $\mu, \nu$
} 
The cocycles independent of the external fields will be denoted as

$$
\Delta^{(G)}=\int d^{6} x Q^{(G)}(x) .
$$

The condition of $\mathcal{S}_{\Sigma}^{0}$-invariance leads to the following set of descent equations:

$$
\mathcal{S}_{\Sigma}^{0} Q_{\left[\mu_{1} . . \mu_{k}\right]}^{(G+k)}=\partial^{\lambda} Q_{\left[\mu_{1} . . \mu_{k} \lambda\right]}^{(G+k+1)}
$$

with $k=0, . ., 6$ and, since the dimension of the space-time is equal to $6, Q_{\left[\mu_{1} . \mu_{7}\right]}^{(G+7)}=0$.

The dimension and ghost number of the fields forbid any possible non-trivial solution in the sector $G=1$, implying the absence of anomaly in the theory.

In the sector $G=0$, the most general solution for the top level of the descent equations reads

$$
Q^{(0)}=\Omega\left(\partial_{[\rho} A_{\mu \nu]}, \bar{a}\right),
$$

where $\Omega\left(\partial_{[\rho} A_{\mu \nu]}, \bar{a}\right)$ is a polynomial depending on $\partial_{[\rho} A_{\mu \nu]}$, with coefficients as arbitrary funcions of $\bar{a}$.

The condition of invariance under the full operator $\mathcal{S}_{\Sigma}$, the constraints (3.13) and (3.14) being taken into account, leads to

$$
\Delta^{(0)}=\Sigma_{\text {inv }},
$$

where $\Sigma_{\text {inv }}$ is the invariant action (3.1). However, the latter is a trivial cocycle:

$$
\begin{aligned}
\Sigma_{\mathrm{inv}}= & \frac{1}{2} \mathcal{S}_{\Sigma} \int d^{6} x\left(\frac{1}{2} A_{\mu \nu}^{*} A^{\mu \nu}-\alpha_{\mu}^{*} \alpha^{\mu}-\phi_{\mu}^{*} \phi^{\mu}+\alpha^{*} \alpha+\omega^{*} \omega+\phi^{*} \phi\right. \\
& \left.-\bar{\alpha}^{\mu} \partial^{\nu} A_{\mu \nu}-\bar{\phi}^{\mu} A_{\mu \nu} \partial^{\nu} a-\bar{\phi}^{\prime} \partial^{\mu} a \phi_{\mu}-\bar{\alpha} \partial^{\mu} \alpha_{\mu}-\bar{\phi} \partial^{\mu} \phi_{\mu}\right) .
\end{aligned}
$$

As a conclusion, the PST model in $D=6$ is finite up to non-physical renormalizations.

\section{The PST model in $\mathrm{D}=4$}

A $p$-form gauge potential, with non-vanishing $(p+1)$-form self-dual field strength in a Minkowski space-time, can only exist in dimensions $D=2(p+1)$ for $p$ even, what restricts the relevant dimensions to $2,6,10, \ldots$

In order to cross over this restriction and define a covariant duality symmetric action in $D=4$, the PST recipe [13] is to introduce two gauge potentials $A_{\mu}^{a}, a=1,2$, the self-duality condition being now a duality relation between the two field strengths $F_{\mu \nu}^{a}$ of the theory. 
The PST action in $D=4$ is

$$
\Sigma_{\mathrm{inv}}=\int d^{4} x\left(-\frac{1}{8} F^{a \mu \nu} F_{\mu \nu}^{a}-\frac{1}{4(\partial a)^{2}} \partial^{\mu} a \mathcal{F}_{\mu \nu}^{a} \mathcal{F}^{a \nu \rho} \partial_{\rho} a\right)
$$

where $F_{\mu \nu}^{a} \equiv \partial_{\mu} A_{\nu}^{a}-\partial_{\nu} A_{\mu}^{a}$ is the field strength of the gauge potential $A_{\mu}^{a}, a$ is a scalar auxiliary field and

$$
\mathcal{F}_{\mu \nu}^{a} \equiv \mathcal{L}^{a b} F_{\mu \nu}^{b}-\frac{1}{2} \epsilon_{\mu \nu \rho \sigma} F^{a \rho \sigma}=\frac{1}{2} \epsilon_{\mu \nu \rho \sigma} \mathcal{L}^{a b} \mathcal{F}^{b \rho \sigma}
$$

where $\mathcal{L}$ is the antisymmetric $(2 \times 2)$-matrix with $\mathcal{L}^{12}=1$.

The action (4.1) has the following symmetries (we only display the non-zero variations):

$$
\begin{aligned}
& \delta_{I} A_{\mu}^{a}=\partial_{\mu} \alpha^{a}, \\
& \delta_{I I} A_{\mu}^{a}=\phi^{a} \partial_{\mu} a, \\
& \delta_{I I I} A_{\mu}^{a}=\beta \hat{B}_{\mu}^{a}, \quad \delta_{I I I} a=\beta, \quad \text { with } B_{\mu}^{a} \equiv \frac{\mathcal{F}_{\mu \nu}^{a} \partial^{\nu} a}{(\partial a)^{2}} \text { and } \hat{B}_{\mu}^{a}=\mathcal{L}^{a b} B_{\mu}^{b}, \\
& \delta_{I V} A_{\mu}^{a}=f_{\mu}^{a}(a), \quad \text { with } f_{\mu}^{a}(a) \text { an arbitrary function of } a .
\end{aligned}
$$

We again must require $\partial^{\mu} a \partial_{\mu} a \neq 0$ and so split $a(x)$ in $\bar{a}(x)$ and $a^{\prime}(x)$ according to (2.4).

The set of local symmetries in (4.3) leads to the following BRST transformations :

$$
\begin{aligned}
& s A_{\mu}^{a}=\partial_{\mu} \alpha^{a}+\phi^{a} \partial_{\mu} a+\beta \hat{B}_{\mu}^{a}, \\
& s a^{\prime}=\beta, \\
& s \beta=0, \\
& s \alpha^{a}=-\beta \phi^{a}, \\
& s \phi^{a}=\frac{\beta}{(\partial a)^{2}}\left(\partial^{\mu} a \partial_{\mu} \phi^{a}-\hat{B}_{\mu}^{a} \partial^{\mu} \beta\right),
\end{aligned}
$$

where $\alpha^{a}, \phi^{a}$ and $\beta$ are now Faddeev-Popov ghosts.

Following the same steps as before, we write down the total action as

$$
\Sigma=\Sigma_{\mathrm{inv}}+\Sigma_{\mathrm{gf}}+\Sigma_{\mathrm{ext}}
$$

with $\Sigma_{\text {inv }}$ given in (4.1) and

$$
\begin{aligned}
& \Sigma_{\mathrm{gf}}=s \int d^{4} x\left(\bar{\alpha}^{a} \partial^{\mu} A_{\mu}^{a}+\bar{\phi}^{a} A_{\mu}^{a} \partial^{\mu} a+\bar{\beta} a^{\prime}\right), \\
& \Sigma_{\mathrm{ext}}=\int d^{4} x\left(A_{\mu}^{* a} s A^{a \mu}+\alpha^{* a} s \alpha^{a}+\phi^{* a} s \phi^{a}\right),
\end{aligned}
$$


where we have introduced the external fields coupled to the non-linear BRST transformations, as well as antighosts and Lagrange multipliers, transforming as

$$
s \bar{C}=\Pi, \quad s \Pi=0,
$$

with $\bar{C}=\left(\bar{\alpha}^{a}, \bar{\phi}^{a}, \beta\right)$ and $\Pi=\left(\pi^{a}, \rho^{a}, b\right)$.

The dimension and ghost number of all fields are displayed below in Table 5 .

\begin{tabular}{|c||c|c|c|c|c|c|c|c|c|c|c|c|c|c|c|}
\hline & $A_{\mu}^{a}$ & $a^{\prime}$ & $\beta$ & $\alpha^{a}$ & $\phi^{a}$ & $\bar{\beta}$ & $b$ & $\bar{\alpha}^{a}$ & $\pi^{a}$ & $\bar{\phi}^{a}$ & $\rho^{a}$ & $A_{\mu}^{* a}$ & $\alpha^{* a}$ & $\phi^{* a}$ & $s$ \\
\hline \hline$d$ & 1 & -1 & -1 & 0 & 1 & 5 & 5 & 2 & 2 & 3 & 3 & 3 & 4 & 3 & 0 \\
\hline$\Phi \Pi$ & 0 & 0 & 1 & 1 & 1 & -1 & 0 & -1 & 0 & -1 & 0 & -1 & -2 & -2 & 1 \\
\hline
\end{tabular}

Table 5: dimension $d$ and ghost number $\Phi \Pi$.

The Slavnov-Taylor identity obeyed by the total classical action (4.5) is found to be

$$
\begin{aligned}
\mathcal{S}(\Sigma)= & \int d^{4} x\left(\frac{\delta \Sigma}{\delta A_{\mu}^{* a}} \frac{\delta \Sigma}{\delta A^{a \mu}}+\frac{\delta \Sigma}{\delta \alpha^{* a}} \frac{\delta \Sigma}{\delta \alpha^{a}}+\frac{\delta \Sigma}{\delta \phi^{* a}} \frac{\delta \Sigma}{\delta \phi^{a}}+\right. \\
& \left.+\beta \frac{\delta \Sigma}{\delta a^{\prime}}+\pi^{a} \frac{\delta \Sigma}{\delta \bar{\alpha}^{a}}+\rho^{a} \frac{\delta \Sigma}{\delta \bar{\phi}^{a}}+b \frac{\delta \Sigma}{\delta \bar{\beta}}\right)=0
\end{aligned}
$$

which leads to the following nilpotent linearized Slavnov-Taylor operator :

$$
\begin{aligned}
\mathcal{S}_{\Sigma}= & \int d^{4} x\left(\frac{\delta \Sigma}{\delta A_{\mu}^{* a}} \frac{\delta}{\delta A^{a \mu}}+\frac{\delta \Sigma}{\delta A^{a \mu}} \frac{\delta}{\delta A_{\mu}^{* a}}+\frac{\delta \Sigma}{\delta \alpha^{* a}} \frac{\delta}{\delta \alpha^{a}}+\frac{\delta \Sigma}{\delta \alpha^{a}} \frac{\delta}{\delta \alpha^{* a}}+\right. \\
& \left.+\frac{\delta \Sigma}{\delta \phi^{* a}} \frac{\delta}{\delta \phi^{a}}+\frac{\delta \Sigma}{\delta \phi^{a}} \frac{\delta}{\delta \phi^{* a}}+\pi^{a} \frac{\delta}{\delta \bar{a}^{a}}+\rho^{a} \frac{\delta}{\delta \bar{\phi}^{a}}+b \frac{\delta}{\delta \bar{\beta}}\right) .
\end{aligned}
$$

The total classical action (4.5) obeys again strong constraints, which can be extended to the quantum level and are important in the study of the cohomology of $\mathcal{S}_{\Sigma}$. The integrated antighost equation for $\alpha$, the integrated equation of motion for $a^{\prime}$ and the constraint between $a^{\prime}$ and $\bar{a}$ are respectively given by

$$
\begin{aligned}
& \int d^{4} x \frac{\delta \Sigma}{\delta \alpha^{a}}=0, \\
& \int d^{4} x \frac{\delta \Sigma}{\delta a^{\prime}}=\int d^{4} x b, \\
& \left(\frac{\delta}{\delta a^{\prime}}-\frac{\delta}{\delta \bar{a}}\right) \Sigma=b .
\end{aligned}
$$

The cohomology of $\mathcal{S}_{\Sigma}$ can be suitably analyzed by expanding it first according to a counting operator like (2.28), acting on all fields, and then by expanding the lowest order contribution of this series in powers of $\phi^{a}$ only. Following exactly the same procedure 
as in the cases $D=2$ and $D=6$ the cohomology of the lowest contribution of the last expansion (in powers of $\phi^{a}$ ) is shown to be empty in the sector with ghost number 1, implying the absence of anomaly. For ghost number 0 we find, at lowest order, the quadratic part of the invariant action (4.1), which corresponds to the full invariant action for the exact $\mathcal{S}_{\Sigma}$ operator. However, this term again is $\mathcal{S}_{\Sigma}$-trivial:

$$
\Sigma_{\text {inv }}=\frac{1}{2} \mathcal{S}_{\Sigma} \int d^{4} x\left(A_{\mu}^{* a} A^{a \mu}-\alpha^{* a} \alpha^{a}-\phi^{* a} \phi^{a}-\bar{\alpha}^{a} \partial^{\mu} A_{\mu}^{a}-\bar{\phi}^{a} A_{\mu}^{a} \partial^{\mu} a\right),
$$

hence the model is finite.

\section{Conclusions}

We have shown, by a purely algebraic analysis independent of any regularization scheme, the absence of gauge anomaly and the finiteness of the PST model - up to possible singularities absorvable by local field redefinitions - in two, four and six dimensions. Both results were established by proving that the BRST cohomology is trivial in the sectors with ghost numbers 0 and 1 . We believe it is possible to extend this proof to all dimensions for which the PST model can be defined. This is a topic we still intend to exploit.

Another point worth to be noticed concerns the implementation of the self-duality constraint at the quantum level. As said before it can be obtained at the classical approximation by means of the equation of motion of $\phi$ and the global symmetry present in the PST model - e.g. given by the transformation (2.3), in the 2-dimensional case. However, the implemention of this argument at the quantum regime is still an open problem. In particular, the validity of the global symmetry has not been shown yet. This kind of discussion remains for future work.

\section{Acknowledgements}

We would like to thank Alexandre de Mello Delpupo for his participation to an early stage of this work. Olivier Piguet is very grateful to the DCP of the Centro Brasileiro de Pesquisas Físicas (CBPF), as well as to the Universidade Católica de Petrópolis, for various invitations during which part important of this work was done. Oswaldo M. Del Cima would like to express his gratitude to J.A. Helayël-Neto and his colleagues at GFTUCP for the warm hospitality and friendship. He dedicates this work to his wife, Zilda Cristina, to his daughter, Vittoria, and to his son, Enzo.

\section{References}


[1] D.J. Gross, J.A. Harvey and E. Martinec Phys.Rev.Lett. 54 (1985) 502.

[2] C.G. Callan, J.A. Harvey and A. Strominger, Nucl.Phys. B367 (1991) 60; E. Witten, J.Geom.Phys. 22 (1997) 103 (hep-th/9610234) and JHEP 0005 (2000) 032 (hep-th/9912279).

[3] P.S. Howe, G. Sierra and P.K. Towsend, Nucl.Phys. B221 (1983) 331;

L.J. Romans Nucl.Phys. B276 (1986) 71;

E. Bergshoeff, E. Sezgin and A. Van Proeyen, Class.Quant.Grav. 16 (1999) 3193 (hep-th/9904085).

[4] X.G. Wen, Phys.Rev.Lett. 64 (1990) 2206.

[5] J.V. Jose, L.P. Kadanoff, S. Kirkpatrick and D. Nelson, Phys.Rev. B16 (1977) 1217; P.B. Wiegmann, J.Phys. C11 (1978) 1583; D. Boyanovsky and R. Holman, Nucl.Phys. B332 (1990) 641;

A. Tseytlin and P. West, Phys.Rev.Lett. 65 (1990) 541.

[6] R. Floreanini and R. Jackiw, Phys.Rev.Lett. 59 (1987) 1873.

[7] M. Henneaux and C. Teitelboim, Phys.Lett. B206 (1988) 650.

[8] J.H. Schwarz and A. Sen, Nucl.Phys. B411 (1994) 35 (hep-th/9304154).

[9] W. Siegel, Nucl.Phys. B238 (1984) 307.

[10] B. McClain, Y.S. Wu and F. Yu Nucl.Phys. B343 (1990) 689.

[11] C. Wotzasek, Phys.Rev.Lett. 66 (1991) 129.

[12] I. Martin and A. Restuccia, Phys.Lett. B323 (1994) 311;

F.P. Devecchi and M. Henneaux, Phys.Rev. D54 (1996) 1606 (hep-th/9603031).

[13] P. Pasti, D. Sorokin and M. Tonin, Phys.Lett. B352 (1995) 59 (hep-th/9503182), Phys.Rev. D52 (1995) R4277 (hep-th/9506109), Leuven Notes in Mathematical and Theoretical Physics, (Leuven University Press), Series BV6 (1996) 167 (hepth/9509052).

[14] P. Pasti, D. Sorokin and M. Tonin, Phys.Rev. D55 (1997) 6292 (hep-th/9611100).

[15] C. Imbimbo and A. Schwimmer, Phys.Lett. B193 (1987) 455;

C.M. Hull, Phys.Lett B206 (1988) 234;

J.M.F. Labastida and M. Pernici, Nucl.Phys. B297 (1988) 557;

L. Mezincescu and R.I. Nepomechie, Phys.Rev. D37 (1988) 3067.

[16] K. Lechner, Nucl.Phys. B537 (1999) 361 (hep/th 9808025). 
[17] I. Bandos, K. Lechner, A. Nurmagambetov, P. Pasti, D. Sorokin and M. Tonin, Phys.Rev.Lett. 78 (1997) 4332 (hep-th/9701149), Phys.Lett. B408 (1997) 135 (hep$\operatorname{th} / 9703127)$.

[18] K. Lechner, Fortsch.Phys. 47 (1999) 209 (hep-th/9712231).

[19] A. Ilha and C. Wotzasek, (hep-th/0004182);

R. Banerjee and C. Wotzasek, (hep-th/0005010).

[20] O. Piguet and S.P. Sorella, "Algebraic Renormalization", Lecture Notes in Physics, m28, Springer-Verlag (Berlin - Heidelberg), 1995.

[21] A. Maznytsia, C.R. Preitschopf and D. Sorokin, Nucl.Phys. B539 (1999) 438 (hepth/9805110).

[22] I.A. Batalin and G.A. Vilkovisky, Phys.Rev. D28 (1983) 2567.

[23] A. Blasi and N. Maggiore, Class.Quant.Grav. 10 (1993) 37 (hep-th/9207008);

R. Leitgeb, M. Schweda and H. Zerrouki, Nucl.Phys. B542 (1999) 425 (hepth/9904204).

[24] J.H. Lowenstein, Phys.Rev. D4 (1971) 2281, Comm.Math.Phys. 24 (1971) 1;

Y.M.P. Lam, Phys.Rev. D6 (1972) 2145, Phys.Rev. D7 (1973) 2943;

T.E. Clark and J.H. Lowenstein, Nucl.Phys. B113 (1976) 109.

[25] J. Dixon, Comm.Math.Phys. 139 (1991) 495;

F. Brandt, N. Dragon and M. Kreuzer, Phys.Lett. B231 (1989) 263, Nucl.Phys. B332 (1990) 224.

[26] C. Becchi, A. Blasi, G. Bonneau, R. Collina and F. Delduc, Comm.Math.Phys. 120 (1988) 121.

[27] G. Barnich, F. Brandt and M. Henneaux, Phys.Rept. 338 (2000) 439 (hepth/0002245). 